\title{
The lengthening effect revisited: A reply to Prinzmetal and Wilson (1997) and Masin (1999)
}

\author{
YEHOSHUA TSAL \\ Tel Aviv University, Tel Aviv, Israel \\ LILACH SHALEV \\ Open University of Israel, Raanana, Israel \\ and \\ DAN ZAKAY \\ Tel Aviv University, Tel Aviv, Israel
}

\begin{abstract}
In the present study, the lengthening phenomenon (Tsal \& Shalev, 1996), namely, the increase in perceived length of unattended lines, was reexamined in light of criticisms by Prinzmetal and Wilson (1997) and Masin (1999). Prinzmetal and Wilson suggested that the effect was not due to attentional factors but to the spatial interaction between the attended line and the cue used to direct attention. We have replicated the lengthening effect when both attended and unattended lines are preceded by cues at a nearby location, showing that the effect is not caused by spatial cues per se, but instead reflects an inherent property of the attentional system. Masin argued that the lengthening effect is not robust, because it occurs for some but not for all participants. In the present study, the lengthening effect was highly reliable, occurring for each participant for a variety of line lengths.
\end{abstract}

In a recent study (Tsal \& Shalev, 1996), we demonstrated a directional effect of attention on length perception. Namely, we observed a systematic tendency to perceive a small vertical line as being longer when unattended than when attended. We explained this phenomenon by proposing that the visual field consists of a grid of small units termed attentional receptive fields (ARFs). When a stimulus appears within the boundaries of a unit, this unit signals its entire length to a central processor. That is, there is no spatial resolution within an ARF, and each unit operates on the basis of an all-or-none principle. In accordance with the resolution theory of visual attention (Tsal, Meiran, \& Lamy, 1995) and with empirical findings by Cohen and Ivry (1991), we further proposed that the unattended visual field is composed of larger ARFs than is the attended field. When a line appears in the visual field, its ends cross the boundaries of adjacent units in most cases, so that these adjacent units are also activated. Because ARFs composing the unattended field are larger than those composing the attended field, an unattended line is systematically perceived as being longer than an attended one. The notion that attention improves spatial resolution is consistent with Yeshurun and Carrasco (1998, 1999, 2000) and Carrasco, Williams, and Yeshurun (2002),

We are grateful to Ray Klein, Glenn Wylie, Anne Hillstrom, Asher Cohen, and an anonymous reviewer for their helpful comments on an earlier draft of this article. Correspondence should be addressed to Y. Tsal, Department of Psychology, Tel Aviv University, Ramat Aviv, Tel Aviv 69978, Israel (e-mail: jehoshua@freud.tau.ac.il). who used a variety of perceptual tasks and showed that attention improves the quality of stimulus representation and that such enhancement results from fine spatial resolution.

The purpose of the present experiments was to reexamine this lengthening phenomenon in light of two recent studies questioning its validity (Prinzmetal \& Wilson, 1997) and consistency (Masin, 1999). Prinzmetal and Wilson argued that the effect was due to the spatial interaction between the cues used to direct attention and the stimulus line, rather than to the operation of attention per se. Although Masin replicated the lengthening effect in one experiment, he argued that the effect is not reliable, because it occurred for some but not all participants in a second experiment. It is important to note that the three studies involved three different manipulations of attention: cuing (Tsal \& Shalev, 1996), dual task (Prinzmetal \& Wilson, 1997), and selective preparation (Masin, 1999). However, the arguments made by Prinzmetal and Wilson (1997) and by Masin (1999) are of sufficient importance to merit investigation within the framework of the cuing paradigm. Therefore, the purpose of the present experiment was not to replicate the studies of Prinzmetal and Wilson (1997) and Masin (1999), nor was it to assess the lengthening effect under different manipulations of attention. Instead, our objective was to reexamine the lengthening effect with a new version of a cuing paradigm that eliminates the potential problems pointed out by Prinzmetal and Wilson (1997) and by Masin (1999). Accordingly, we used a design that allowed assessing the effects of attention, while controlling for the possible 
confounding effects of the spatial cues, and performed a more detailed analysis to examine the lengthening effect for each participant.

In their first two experiments, Prinzmetal and Wilson (1997) manipulated attention by using a dual task. Participants had to detect a central target and estimate the length of a peripheral line. The central target appeared either simultaneously with the peripheral line or shortly before it. The authors reasoned that in the former condition, fewer attentional resources should be allocated to the line because this condition required participants to attend to two objects at the same time. Contrary to our finding (Tsal \& Shalev, 1996), Prinzmetal and Wilson found no difference in length judgments in the simultaneous versus successive presentation conditions. They concluded that unattended lines are not perceived as being longer than attended ones. In their third experiment, Prinzmetal and Wilson presented the peripheral lines either alone or with spatial cues. However, the cues actually succeeded the presentation of the lines. They found the lines to be perceived as being longer in the blank condition than in the cued condition. On the basis of this finding, Prinzmetal and Wilson argued that the lengthening effect reported in our study was not due to attention, but simply to the presence or absence of cues in the attended and unattended trials, respectively. Note that this conclusion is unwarranted, because Prinzmetal and Wilson did not conduct an exact replication of our study. Indeed, while their experiment showed that the presence of cues could systematically distort length perception, it did not establish that the entire lengthening effect was due to the cues per se.

Masin (1999) presented a line on one side of fixation and a square on the other. In the attended trials, participants were told beforehand where the line would appear. In the unattended trials, they were told where the square would appear and had to judge either the length or the brightness of the line, depending on the color of the square. In the first experiment, Masin confirmed our results by showing that the unattended line was estimated as being longer than the attended one. In the second experiment, he repeated the same procedure but increased the number of trials and used a more detailed analysis for each individual participant. Here, he found the effect to be replicated for some but not all participants. Masin concluded that the effect is not reliable and explained his results by proposing that some participants may have preferred small, and some large, responses, and these responses became smaller and larger, respectively, as uncertainty increased with inattention. These biases could explain the significant lengthening for some participants and significant shortening for other participants with inattention.

\section{EXPERIMENT 1}

In order to investigate whether the lengthening effect observed in our previous study was due to reduced attention or to the presence or absence of spatial cues, as suggested by Prinzmetal and Wilson (1997), we created a de- sign in which both attended and unattended lines were preceded by spatial cues. The participants were required to estimate the length of a line that appeared in one of five (one central and four diagonal peripheral) possible positions. The participants were tested under two conditions: the focused attention condition, in which a single precue directed attention to the designated stimulus location, and the distributed attention condition, in which all five locations were precued. This manipulation allowed us to assess the effects of attention on length perception by comparing length estimates when attention is focused on the stimulus location versus when attention is distributed over a relatively large area of the visual field. In order to address Prinzmetal and Wilson's concerns, the stimulus line was preceded by a spatial cue at a nearby location under both conditions, such that the attention manipulation was not contaminated by the differences in spatial cues. Furthermore, in order to address Masin's arguments, we investigated the reliability of the lengthening effect by looking at it for a variety of line lengths for each individual participant.

\section{Method}

Participants. Ten Tel Aviv University undergraduates participated in the experiment as part of a course requirement. All had normal or corrected-to-normal vision.

Stimuli and Procedure. Stimulus presentation and data collection were controlled by an IBM PC/AT with a VGA graphic display. A chinrest was used to stabilize viewing distance at $50 \mathrm{~cm}$, so that $1 \mathrm{~cm}$ on the display represented $1.15^{\circ}$ of visual angle. The stimulus was one of five possible white vertical lines $\left(20 \mathrm{~cd} / \mathrm{m}^{2}\right)$ appearing on a dark background $\left(1 \mathrm{~cd} / \mathrm{m}^{2}\right)$. The shortest line subtended $0.58^{\circ}$, and each successive line was incremented by $0.23^{\circ}$, so that the longest line subtended $1.5^{\circ}$ of visual angle. The line appeared equally frequently and in random order in one of five possible positions: at the center or at each corner of an imaginary square, the side of which subtended $3.2^{\circ}$ of visual angle. The participants were tested under two conditions. In the focused attention condition, a precue indicated the position of the subsequent line with $100 \%$ validity. In the distributed attention condition, all five positions were precued. The cue was a white open circle subtending $0.6^{\circ}$ of visual angle. It was placed $0.6^{\circ}$ closer to the center for the peripheral lines and $0.5^{\circ}$ to the right or left of the central line. Each line length was presented 30 times. The five line lengths were randomly intermixed, and each was randomly assigned to the various possible positions. Each trial consisted of the following sequence of events (see Figure 1). First, a small fixation cross was presented for $1,000 \mathrm{msec}$. Then, the cross disappeared, and after $150 \mathrm{msec}$, the cue(s) appeared for $50 \mathrm{msec}$. Then, following an interstimulus interval of $50 \mathrm{msec}$, the target line was presented for $50 \mathrm{msec}$. Thus, the total duration between cue onset and target offset was $150 \mathrm{msec}$. Nine response lines of different length, labeled 1 to 9 , were presented in ascending order from left to right, $500 \mathrm{msec}$ after stimulus offset. The five intermediate lines were the ones actually used as stimuli in the experiment. The four additional response lines were added so as to extend the boundaries of the response scale, in order to minimize biases toward overestimating short lines and underestimating long lines. The response lines remained in sight until the participants responded by pressing the corresponding number on the extended keyboard. Five participants were presented first with the focused attention condition, and five were presented first with the distributed attention condition. Each condition consisted of 150 trials and was preceded by 20 practice trials. 


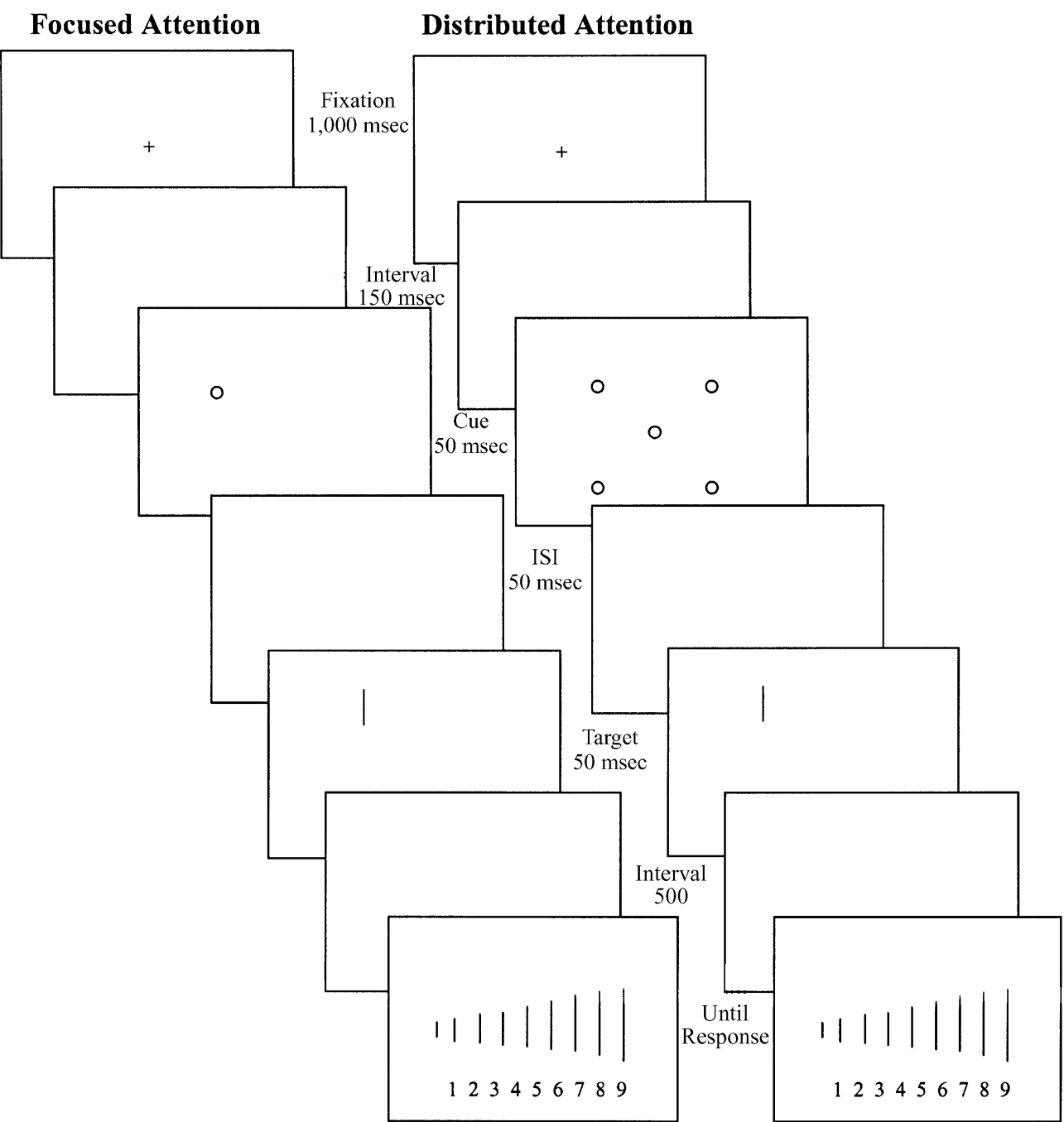

Figure 1. Sequence of events of typical trials in the two attention conditions.

\section{Results and Discussion}

The mean deviation of the perceived length from the objective length for each of the five lines was calculated for each participant. These values for attended (focused attention condition) and unattended (distributed atten- tion condition) lines appearing at the center and at the periphery are presented in Table 1 . Positive and negative numbers indicate that the line was perceived as longer or shorter than its objective length, respectively. As is clear from the Table 1, unattended lines were systematically

Table 1

Mean Deviations in Degrees of Visual Angle From

Objective Length for Attended and Unattended Lines Appearing at the Center and at the Periphery in Experiment 1

\begin{tabular}{|c|c|c|c|c|}
\hline \multirow[b]{2}{*}{ Line Length } & \multicolumn{2}{|c|}{ Central-Appearing Lines } & \multicolumn{2}{|c|}{ Peripheral-Appearing Lines } \\
\hline & Attended & Unattended & Attended & Unattended \\
\hline .58 & -.07 & .11 & -.03 & .28 \\
\hline .81 & -.10 & .11 & -.05 & .27 \\
\hline 1.04 & -.13 & .13 & -.08 & .21 \\
\hline 1.27 & -.17 & .05 & -.09 & .15 \\
\hline 1.50 & -.18 & .03 & -.09 & .13 \\
\hline$M$ & -.130 & .086 & -.068 & .208 \\
\hline
\end{tabular}

Note-Positive values indicate overestimations, and negative values indicate underestimations. 
perceived as being longer than attended lines, and peripheral lines were perceived as being longer than central lines. The table also shows that for all stimulus lengths, overestimation gradually decreased with increased length. These observations were confirmed by statistical analyses.

The mean deviations of perceived length from objective length were submitted to an overall analysis of variance (ANOVA) of attention condition $\times$ eccentricity $\times$ length $\times$ participants. The analysis indicated that the three main effects were highly significant. Unattended lines were perceived as being longer than attended ones $[F(1,9)=52.95, p<.0001]$, peripheral lines were perceived as being longer than central ones $[F(1,9)=9.12$, $p=.014]$, and overestimation decreased as line length increased $[F(4,36)=9.16, p<.0001]$. The interaction of attention and eccentricity was also significant, indicating that the effect of attention was stronger for peripheral than for central lines $[F(1,9)=8.24, p<.05]$. None of the other interactions reached statistical significance.

Two additional ANOVAs on the separate effects of attention and eccentricity on perceived length were used to assess the similarity in their pattern of results. An ANOVA of attention condition $\times$ line length $\times$ participants indicated that the two main effects were highly significant. Shorter lines produced greater overestimates than longer lines $[F(4,36)=8.19, p<.0001]$, and unattended lines were perceived as being longer than attended ones $[F(1,9)=12.67, p<.01]$. The interaction between the two factors was not significant $[F(4,36)=$ 1.53], indicating that the magnitude of the effect of attention was maintained across all line lengths. An additional ANOVA of eccentricity $\times$ line length $\times$ participants showed that the two main effects were highly significant. Shorter lines produced stronger overestimates than longer lines $[F(4,36)=7.64, p<.0001]$ and peripheral lines were perceived as being longer than central lines $[F(1,9)=49.17$, $p<.0001]$. The interaction between the two factors did not reach significance $[F(4,36)=.72]$, indicating that the effect of eccentricity was stable across all line lengths. Clearly, attention and eccentricity produced parallel patterns of results.

Table 1 also shows that the two longest central lines were, in fact, estimated more accurately under the distributed attention condition than under the focused attention condition. This does not reflect a reduced perceptual accuracy with attention, but rather a bias inherent in the measurement scale. As we observed in our previous study (Tsal \& Shalev, 1996; see also Klein, Wylie, \& Briand, 1996), there is a tendency to underestimate the long lines (thus increasing underestimation and reducing overestimation) because they are bound by the upper limit of the judgment scale. This bias, coupled with the increase in perceived length of unattended lines naturally produces more accurate estimates for the long unattended lines than for the long attended ones.

Table 2 shows that the effect of attention was extremely reliable because it was obtained for each participant for
Table 2

Mean Differences in Length Estimates Between Unattended and Attended Lines (Unattended - Attended) in Degrees of Visual Angle for Each Line Length for Each Participant in Experiment 1

\begin{tabular}{cccccc}
\hline & \multicolumn{5}{c}{ Line Length } \\
\cline { 2 - 6 } Participant & .58 & .81 & 1.04 & 1.27 & 1.50 \\
\hline 1 & .146 & .228 & .202 & .201 & .166 \\
2 & 1.057 & .941 & .905 & .730 & .658 \\
3 & .107 & .179 & .327 & .277 & .133 \\
4 & .197 & .267 & .179 & .140 & .087 \\
5 & .130 & .298 & .328 & .302 & .273 \\
6 & .240 & .298 & .312 & .315 & .358 \\
7 & .125 & .138 & .158 & .092 & .253 \\
8 & .107 & .089 & .173 & .037 & -.059 \\
9 & .322 & .215 & .335 & .279 & .206 \\
10 & .093 & .030 & .106 & .000 & .107 \\
\hline
\end{tabular}

Note-Positive numbers indicate that unattended lines were perceived as being longer than attended ones. The line lengths are for Lines 1-5, from left to right.

almost all line lengths. This table presents the difference in length estimates between unattended and attended lines for each line and each participant, with positive numbers indicating that the unattended line was perceived as being longer than the attended one. For Participant 8, Line 5 was perceived as being longer when it was attended than when it was unattended and for Participant 10, attention had no effect on the perception of Line 4. All other lines were perceived as being longer when unattended than when attended for each participant.

\section{EXPERIMENT 2}

One may possibly argue that another difference between the two conditions was responsible for the results. Although in both conditions, a single cue occupied a location near the stimulus, the cue itself could have been more salient in the focused attention condition when it appeared by itself than in the distributed attention condition when four additional cues appeared in different locations in the visual field. In order to rule out this possibility, we performed an additional experiment that showed that cue salience in itself does not influence length judgments.

\section{Method}

Participants. Nine Tel Aviv University undergraduates participated in the experiment as part of a course requirement. All had normal or corrected-to-normal vision.

Stimuli and Procedure. The stimuli and procedure were identical in all respects to the distributed attention condition of Experiment 1 , except that one of the five dots was salient. Five participants were presented with four white cues and one red cue, and 4 were presented with four red cues and one white cue. The salient dot was randomly assigned to one of the five possible positions but was not informative of the location of the subsequent stimulus (and the participants were informed accordingly).

\section{Results and Discussion}

The mean deviation of the perceived length from the objective length was calculated for each participant. These 
Table 3

Mean Deviations in Degrees of Visual Angle From Objective Length for the Two Types of Displays in Experiment 2

\begin{tabular}{lccccc}
\hline & \multicolumn{2}{c}{ Central-Appearing Lines } & & \multicolumn{2}{c}{ Peripheral-Appearing Lines } \\
\cline { 2 - 3 } \cline { 5 - 6 } Display Type & Salient Cue & Nonsalient Cue & & Salient Cue & Nonsalient Cue \\
\hline Four red, one white & -.141 & -.163 & & -.073 & -.091 \\
Four white, one red & -.138 & -.132 & & -.038 & -.027 \\
$M$ & -.140 & -.148 & & -.056 & -.059 \\
\hline Note-Negative values indicate underestimations. & &
\end{tabular}

values are presented in Table 3. As the results show, all lines were slightly underestimated. This deviation is similar to the one obtained in previous studies (e.g., Prinzmetal \& Wilson, 1997; Tsal \& Shalev, 1996) and may possibly result from the brief presentation conditions.

Most importantly, as is clear from Table 3, lines appearing near a salient cue were not perceived as being shorter than those appearing near a nonsalient cue. In fact, there was a slight tendency in the opposite direction, but this effect was not significant $[F(1,8)=0.591]$. Thus, cue salience in itself does not influence length judgments. The analysis indicated that the only significant effect was that of eccentricity; as in Experiment 1 and in our previous study (Tsal \& Shalev, 1996), peripheral lines were perceived as being longer than central ones $[F(1,8)=19.8$, $p=.002]$. As Table 3 shows, lines presented near a white cue (lines presented near a salient cue in the four-red, onewhite displays and lines presented near a nonsalient cue in the four-white, one-red displays) were estimated as being slightly longer than those presented near a red cue (lines presented near a salient cue in the four-white, one-red displays and lines presented near a nonsalient cue in the fourred, one-white displays). However, an additional analysis comparing responses with the above display types indicated that this effect was not significant $[F(1,8)=2.59$, $p=.146]$, nor did it interact with eccentricity $[F(1,8)=$ $0.021, p=.889]$. Thus, the local contrast in the proximity of the line had no effect on length estimates.

\section{GENERAL DISCUSSION}

The present findings provide a strong replication of our previous study (Tsal \& Shalev, 1996). Unattended lines were systematically perceived as being longer than attended ones.

The results of the present study cannot be reconciled with Prinzmetal and Wilson's (1997) interpretation. Indeed, they cannot be attributed to the spatial interaction between the attended line and the spatial cue used to direct attention, because both attended and unattended lines were preceded by a cue at a nearby location. The failure of Prinzmetal and Wilson to obtain similar findings could possibly result from a relatively weak manipulation of attention, because in both their simultaneous and successive conditions the line appeared at an unexpected peripheral location. In contrast, we compared length judgments between a condition where attention was either distributed (the present study) or misdirected (Tsal \& Shalev, 1996) and a condition where attention was directed to the very location occupied by the target line. Furthermore, Prinzmetal and Wilson used a continuous scale method to assess length judgments. A standard response line followed the test line and was adjusted by moving the mouse up and down, making the line appear longer or shorter, respectively. However, one should note that production of the response is likely to create noise and possibly distort the original impression of the stimulus. The participant began with a partly arbitrary length that needed to be either gradually (Experiments 1 and 3 ) or categorically (Experiment 2) adjusted toward the final response. During this process, the participant experienced a range of lengths, intervening between the stimulus presentation and the final response, which could obviously bias his or her response of length. This method is therefore inferior to simultaneously presenting several response categories of lengths. Moreover, it could increase variability that minimizes possible perceptual differences between attended and unattended lines.

The lengthening effect obtained in our study was extremely robust since, except for two judgments, it was obtained for all five lines for each participant (see Table 2). Thus, contrary to Masin's (1999) arguments, the phenomenon is reliable. Note, however, that Masin presented only a single line, $50 \mathrm{~mm}$ long, repeatedly throughout the experiment. Clearly, such a procedure is likely, if anything, to increase perceptual certainty, thereby minimizing any possible perceptual differences between the two attention conditions.

Interestingly, as in Tsal and Shalev (1996), reduced attention and eccentricity produced parallel lengthening effects. Indeed, diverting attention from the location of the stimulus resembled placing it in the periphery, suggesting that the unattended field may be characterized by a loss of resolution. This finding is consistent with the conclusions of Yeshurun and Carrasco (1998, 1999, 2000) and supports our hypothesis (Tsal \& Shalev, 1996) that the visual field consists of ARFs, which are relatively large in unattended areas and within which there is no spatial resolution.

Three additional studies assess the effects of attention on length perception. Two of them reported that attention, in fact, increased perceived line length. However, both studies suffer from methodological limitations. Fraisse, Ehrlich, and Vurpillot (1956) manipulated atten- 
tion by simply instructing participants to look at one of two horizontal lines, hence, confounding attention with eye fixation. In a recent study, Masin (2003) reported that attention increases perceived length of vertical and horizontal peripheral lines. Masin used a central endogenous cue with a total ISI between cue onset and stimulus offset of only $170 \mathrm{msec}$. While such durations are sufficient to attract attention by abrupt exogenous cues, they are far too short to direct attention voluntarily. Indeed, attention driven by endogenous cues is much slower, rising gradually to a maximum of about $300-400 \mathrm{msec}$ after cue onset. Therefore, the effects reported in Masin's (2003) study are probably not produced by the manipulation of attention.

Klein et al. (1996) presented four diagonal lines around fixation and manipulated attention by using either valid, neutral, or invalid cues. They found that attention only influenced reaction times. Similarly to Prinzmetal and Wilson (1997), they found no directional effects of attention on length perception. In contrast to Prinzmetal and Wilson, they did not observe any effect of attention on accuracy of length judgments. Klein et al. suggested that the conflict between their results and those of Prinzmetal and Wilson may be attributed to the fact that the dual task (Prinzmetal \& Wilson) and directing attention to location (Klein et al.) are qualitatively different phenomena. They further suggested that the conflict between their results and those of Tsal and Shalev (1996) may be due to the fact that in the latter study the lines were not terminated. Another possibility is that the reaction time method used by Klein et al. was not sufficiently sensitive to reveal the effects of attention on length judgments. Although speed of responses was not emphasized, the relatively fast responses (below $1 \mathrm{sec}$ ) may have precluded the careful assessment of stimulus magnitude, thus obliterating potential subtle differences between responses to attended and unattended lines.

Whereas the present results provide strong support for the lengthening effect within the present experimental paradigm, the general issue of whether inattention does (Masin, 1999, Experiment 1; Tsal \& Shalev, 1996; present article) or does not (Klein et al., 1996; Masin, 1999,
Experiment 2; Prinzmetal \& Wilson, 1997) increase perceived line length is far from resolved. The discrepant results obtained in these studies could reflect different manipulations of attention, different procedures, or different stimulus displays. A resolution of this issue would clearly require empirical tests entailing comparisons of the various methodologies. Only such comparisons could either provide one definitive answer or alternatively indicate that different manipulations of attention reflect different aspects of attention.

\section{REFERENCES}

Carrasco, M., Williams, E. M., \& Yeshurun, Y. (2002). Covert attention increases spatial resolution with or without masking: Support for signal enhancement. Journal of Vision, 2, 467-479.

Cohen, A., \& Ivry, R. (1991). Density effects in conjunction search: Evidence for a coarse location mechanism of feature integration. Journal of Experimental Psychology: Human Perception \& Performance, 17, 891-901.

FrAisSE, P., Ehrlich, S., \& Vurpillot, E. (1956). Etudes de la centration perceptive par la methode tachistoscopique. Archives de Psychologie, 35, 193-214.

Klein, R. M., Wylie, G., \& Briand, K. (1996). How does covert visual orienting affect absolute judgments of size and duration? Abstracts of the Psychonomic Society, 1, 40.

Masin, S. C. (1999). Attention and estimated line length. Perceptual \& Motor Skills, 88, 831-842.

MASIN, S. C. (2003). Test of the effect of attention on judged length of a line. Perceptual \& Motor Skills, 96, 599-606.

Prinzmetal, W. A., \& Wilson, A. (1997). The effect of attention on phenomenal length. Perception, 26, 193-205.

Tsal, Y., Meiran, N., \& LAMY, D. (1995). Towards a resolution theory of attention. Visual Cognition, 2, 313-330.

Tsal, Y., \& ShaLeV, L. (1996). Inattention magnifies perceived length: The attentional receptive field hypothesis. Journal of Experimental Psychology: Human Perception \& Performance, 22, 233-243.

Yeshurun, Y., \& CARRAsco, M. (1998). Attention improves or impairs visual performance by enhancing spatial resolution. Nature, 396, 7275.

Yeshurun, Y., \& CARRasco, M. (1999). Spatial resolution improves performance in spatial resolution tasks. Vision Research, 39, 293-305.

Yeshurun, Y., \& CARrasco, M. (2000). The locus of the attentional effects of texture segmentation. Nature Neuroscience, $\underline{\mathbf{3}, 622-627 .}$

(Manuscript received February 19, 2003; revision accepted for publication January 14, 2004.) 